\title{
High resolution spectroscopy and emission line imaging of DEM L 71 with XMM-Newton
}

\author{
K. J. van der Heyden ${ }^{1}$, J. A. M. Bleeker ${ }^{1}$, J. S. Kaastra ${ }^{1}$, and J. Vink ${ }^{2,3}$ \\ 1 SRON National Institute for Space Research, Sorbonnelaan 2, 3584 CA Utrecht, The Netherlands \\ 2 Columbia Astrophysics Laboratory, Columbia University, 550 West 1 20th Street, New York, NY 10027, USA \\ ${ }^{3}$ Chandra fellow
}

Received 5 February 2003 / Accepted 15 April 2003

\begin{abstract}
The X-ray emission from the supernova remnant DEM L 71 is measured and analysed using the high-resolution cameras and spectrometers on board XMM-Newton. The spectrum from the outer shell is reproduced very well by two plasma components of $k T_{\mathrm{e}}=0.3$ and $0.8 \mathrm{keV}$. The abundance value from this shell is consistent with the average LMC values. More extreme temperature variations are possibly indicated by spatial variations in the O VII forbidden/resonance line ratio, which could imply that in some regions the plasma is cooling dramatically and recombining. However, an alternative and equally interesting possibility is that the variation in forbidden/resonance ratios is due to resonant scattering, which would reduce resonance line emission along lines of sight with a high O VII column density. The inner region is hotter $\left(k T_{\mathrm{e}}=1.1 \mathrm{keV}\right)$ and shows enhanced $\mathrm{Fe}$ and Si abundances. We estimate the Fe and Si ejecta mass to be $0.7-1.1 M_{\odot}$ and $0.1-0.15 M_{\odot}$, respectively. The morphology, mass estimates and abundances strongly suggest that DEM L 71 is the result of a type Ia explosion, as indicated by previous measurements.
\end{abstract}

Key words. ISM: supernova remnants - ISM: individual objects: DEM L 71 - ISM: abundances - shock waves

\section{Introduction}

DEM L 71 (SNR 0505-67.9) belongs to a class of Supernova Remnants (SNR) that are dominated by hydrogen emission in their optical spectra with virtually no emission from collisionally excited forbidden lines. Analysis of the optical spectrum of DEM L 71 indicates a shock velocity of $300-800 \mathrm{~km} \mathrm{~s}^{-1}$ which corresponds to a shock temperature of $T_{\mathrm{s}}=0.11-0.75 \mathrm{keV}$ (Smith et al. 1994). This information, combined with a radial size of $\sim 10 \mathrm{pc}$, gives an estimated age of $\sim 10^{4}$ years. $A S C A$ spectroscopy revealed that, despite its age, DEM L 71 still showed emission from ejecta material in the form of enhanced Fe abundance (Hughes et al. 1998). The enhanced Fe abundance indicates that DEM L 71 is the result of a type Ia SNe. The recent Chandra Fe-L images (Hughes et al. 2003; see also Fig. 1) also reveal that the remnant exhibits both shell-like $\mathrm{X}$-ray emission and a centrally filled morphology in this energy range.

In this work, we present the X-ray spectra of DEM L 71 measured by the XMM-Newton suite of scientific instruments, i.e. the Reflection Grating Spectrometers (RGS) (den Herder et al. 2001) and the European Photon Imaging Cameras (EPIC) (Turner et al. 2001; Strüder et al. 2001). In particular, we exploit the high dispersion of the RGS and its unique capability to resolve line emission from extended sources to investigate

Send offprint requests to: K. J. van der Heyden, e-mail: K.J.van.der.Heyden@sron.nl the conditions of the X-ray emitting plasma in DEM L 71 in unprecedented detail.

\section{Observation and reduction}

Our analysis is based on XMM-Newton observations of DEM L 71 obtained on 2 April 2001. The observation was performed with the telescope rolled such that the RGS dispersion axis was aligned at $72^{\circ}$, clockwise on the sky from celestial North. A large fraction of the observation is affected by high particle background caused by solar activity. For the EPIC instruments periods of high particle background were rejected based upon the 10-12 keV count rate for the entire field of view. After filtering out these high background periods we are left with a net exposure time of $10 \mathrm{ks}$ for the EPIC instruments. The EPIC instruments were all operated in full window mode with the medium filter in place. Good time intervals (GTI) for the RGS were created based on the count rate in CCD number 9 (it is the one closest to the optical axis of the telescope, therefore the most affected by background flares). After applying the GTI filter we are left with an effective exposure time of $40 \mathrm{ks}$ for the RGS. The spectra were extracted by applying spatial filters to the CCD image while a CCD pulse-height filter was applied to select the $m=-1$ spectral order. All the $X M M-N e w t o n$ data were processed using the Science Analysis System (SAS version 5.3). 


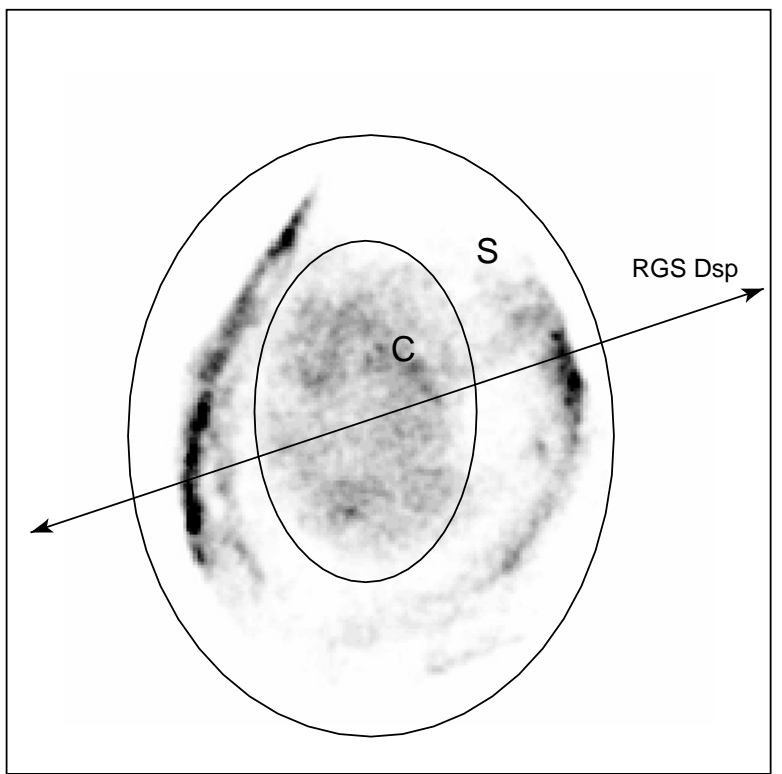

Fig. 1. Chandra image for the approximate energy range $0.7-1.0 \mathrm{keV}$. The regions used for the extraction of the outer shell (S) and central region $(\mathrm{C})$ are indicated. The alignment of the RGS dispersion axis is also indicated.

\section{Analysis and results}

\subsection{Spectra}

The spectral analysis was performed using the SRON SPEX package (Kaastra et al. 1996), which contains the MEKAL atomic database (Mewe et al. 1995) for thermal emission. Our model for the X-ray emission of DEM L 71 consists of several plasma components. For each component, we fit for the volume emission measure $\left(n_{\mathrm{e}} n_{\mathrm{H}} V\right)$, the electron temperature $\left(T_{\mathrm{e}}\right)$, the ionisation age $\left(n_{\mathrm{e}} t\right)$ (Kaastra \& Jansen 1993), the elemental abundances, the redshift of the source, and the column density $N_{\mathrm{H}}$ of absorbing gas along the line of sight. Here, $n_{\mathrm{e}}$ is the electron density, $n_{\mathrm{H}}$ is the hydrogen density, $V$ is the volume of emitting gas and $t$ is the time since the material has been shocked and heated to its current temperature. A distance of $52 \mathrm{kpc}$ to the LMC is assumed (Feast et al. 2002).

The first order RGS spectrum of DEM L 71 is shown in Fig. 2. The spectrum is dominated by emission lines, the most prominent of which are from transitions of highly ionised ions of $\mathrm{C}, \mathrm{O}, \mathrm{Fe}$ and Ne. The $\mathrm{O}$ VIII-L $\alpha$ and $\mathrm{O}$ VII-triplet lines are particularly strong. Weaker, but unambiguous, emission from ions of $\mathrm{N}, \mathrm{Mg}$ and $\mathrm{Si}$ are also present. While the second order RGS spectrum has higher spectral resolution, it does not provide any additional information. The second order spectrum is also of lower statistical quality and was therefore not used.

We fitted the RGS $1 \& 2$ spectra, extracted from the entire source, simultaneously. A response matrix appropriate to the spatial extent of the source was generated as follows. A spatial mask corresponding to the RGS aperture was imposed on the Chandra ACIS image, and the intensity distribution was integrated over the RGS cross-dispersion direction. The resulting profile was convolved with the RGS point source response matrix, generated with the SAS task RGSRMFGEN. We find that one plasma component does not describe the data sufficiently well and that at least two NEI plasma components are needed for obtaining a good fit to the RGS spectrum. A hotter component $\left(k T_{\mathrm{e}} \sim 0.8 \mathrm{keV}\right)$ describes the spectrum below $\sim 20 \AA$, while a cooler component $\left(k T_{\mathrm{e}} \sim 0.2 \mathrm{keV}\right)$ is needed to model the emission from the O VII-triplet through $\mathrm{C}$ VI. When fitting the abundances we used $\mathrm{O}$ as the reference atom and, for technical reasons, kept its abundance fixed to solar. We use $\mathrm{O}$ because it has the strongest emission and would thus give the least uncertainty in the abundance determination. We allowed the abundance of $\mathrm{H}, \mathrm{C}, \mathrm{N}, \mathrm{Ne}, \mathrm{Mg}, \mathrm{Si}$ and Fe to vary with respect to $\mathrm{O}$. The He abundance was pegged to the $\mathrm{H}$ value. The best fit results, given in Table 1, gives a $\chi^{2} /$ d.o.f. 1869/945. We obtain a best fit $N_{\mathrm{H}}=(9.5 \pm 3.1) \times 10^{24} \mathrm{~m}^{-2}$ and a systematic redshift of $380 \pm 120 \mathrm{~km} \mathrm{~s}^{-1}$, which is consistent with the LMC radial velocity $\left(278 \mathrm{~km} \mathrm{~s}^{-1}\right)$. The derived elemental abundances are consistent with the average LMC abundance values. We discuss this more extensively in Sect. 4.2.

The model provides very good fits to the data, though a few discrepancies exist. For the most part, the fit discrepancies in Fig. 2 are a result of the difference between the broad band spatial profile used in the response and the actual spatial profiles of individual emission lines. The images in Fig. 4 and the monochromatic images in Sect. 3.2 show that the remnant's morphology depends strongly on wavelength.

In addition to the spectral features which are present in the RGS spectra, the EPIC spectra also show more prominent emission from $\mathrm{Si}$ and also $\mathrm{S}$. We do not detect any significant $\mathrm{Fe}-\mathrm{K}$ emission nor emission from $\mathrm{Ca}$ and Ar. We extracted and fitted the EPIC-pn spectrum from the entire source. We use the EPIC-pn spectra because they provide the best statistics. As a starting point we used the same model and parameters obtained from the RGS fits. We first allow only the normalisations of the plasma components to vary and subsequently we allowed the absorption, temperature, ionisation age and abundances to vary. The fit results are supplied in Table 1. The derived parameters, except the normalisations, from the fit to the EPIC-pn data remain similar to those obtained from the RGS. The larger normalisations for the EPIC-pn are due to the higher $N_{\mathrm{H}}$ and differences in the RGS and EPIC-pn calibrations (of the order $10 \%)$.

We also extracted and fitted the EPIC-pn spectra from both the outer and central region (see Figs. 1 and 3). The spectrum from the inner region is dominated by a broad emission peak around $0.9 \mathrm{keV}$ from Fe-L. The spectrum from the outer rim is softer and shows more pronounced O-K (O VII and O VIII) and $\mathrm{Mg}-\mathrm{K} \alpha$ lines. We fitted the spectrum extracted from the rim first. We find that this spectrum can not be well described using only one plasma component. A model composed of two NEI plasma components provides a better fit to the data. The fitting procedure was the same as for the full EPIC-pn spectrum. We then fitted the spectrum from the inner region. As a model we used one NEI component to account for emission from the central shell-like structure. In addition to this, we added the same plasma components and parameters as derived from the fits to the outer region. Only the normalisations from these components were allowed to vary. We do this to account for any projected fore/background shell emission. 


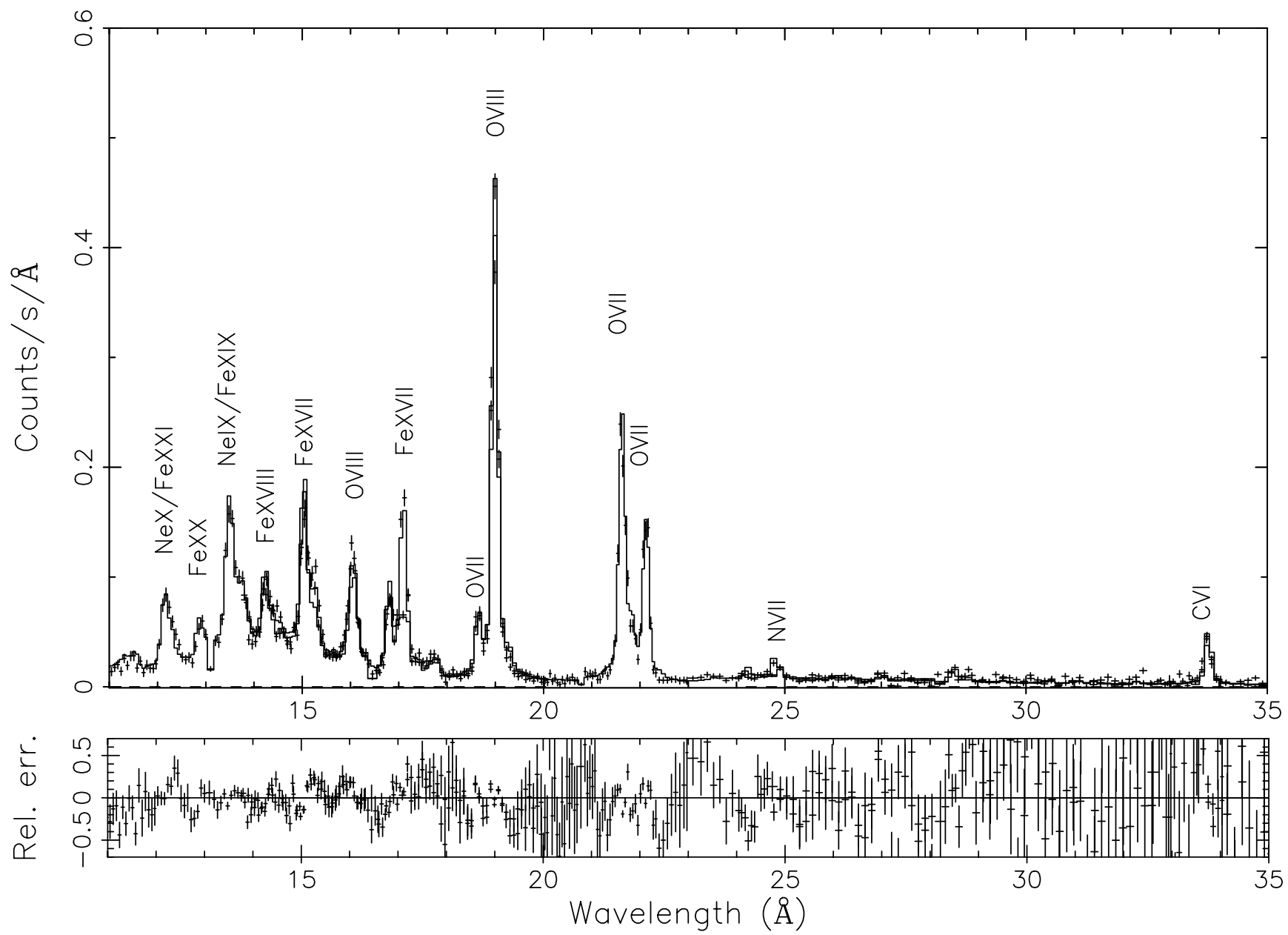

Fig. 2. The first order RGS spectra of DEM L 71 in the range 11-35 ̊. RGS-1 and RGS-2 spectra are overplotted. The solid line represents a best-fit 2 component NEI model $\left(k T_{\mathrm{e}}=0.2\right.$ and $\left.0.8 \mathrm{keV}\right)$. The most prominent line blends are labeled.

The results of the fits are supplied in Table 2. The shell components $\left(k T_{\mathrm{e}} \sim 0.3\right.$ and $\left.0.8 \mathrm{keV}\right)$ have lower temperatures than the central component $\left(k T_{\mathrm{e}} \sim 1.1\right)$. Another significant difference between the two regions is the $\mathrm{Fe} / \mathrm{O}$ and $\mathrm{Si} / \mathrm{O}$ abundance ratio. The outer region has a Fe $/ \mathrm{O} \sim 1.6$ and $\mathrm{Si} / \mathrm{O} \sim 1.5$, while the inner regions has a much higher $\mathrm{Fe} / \mathrm{O} \sim 5.9$ and $\mathrm{Si} / \mathrm{O} \sim 2.3$. The interpretation is discussed in Sect. 4.3.

Some systematic fit residuals are present in the EPIC-pn fits. The most prominent residual is the underestimation of the spectra at $\approx 1.2 \mathrm{keV}$. This is a known problem and is probably due to missing high excitation lines of Fe XVII-XIX in the plasma code (see Brickhouse et al. 2000).

\subsection{Monochromatic images and single ion spectroscopy}

We extracted monochromatic images for various lines to probe variations in temperature and ionisation ages over the remnant and to further investigate the morphological differences between $\mathrm{O}$ and $\mathrm{Fe}$ emission. In order to compare RGS line images to one another one has to take into account an important systematic effect, which is the chromatic magnification that makes long wavelength images more "squashed" than the

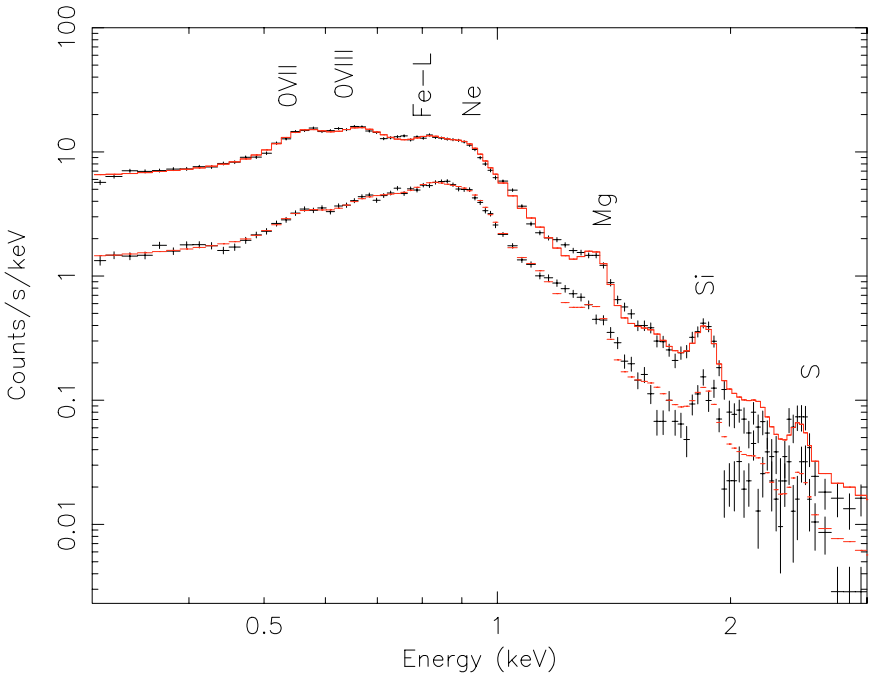

Fig. 3. The DEM L 71 spectrum obtained with the EPIC-pn. The upper curve represents the spectrum from the shell, while the lower curve is from the central region. The solid line represents a best-fit NEI models. The most prominent line blends are labeled.

shorter wavelength images. We correct for this effect, to first order, by using the grating equations. The extent of the source 
Table 1. Best fit parameters for fits to the RGS and EPIC-pn spectra, extracted from the entire remnant. The abundance values are all normalised to $\mathrm{O}$ and relative to solar (Anders \& Grevesse 1989). Note that $\mathrm{C}$ and $\mathrm{N}$ emission can not be determined from the EPIC-pn data, while the S-K emission is too weak to be detected by the RGS.

\begin{tabular}{|l|c|c|c|c|}
\hline \hline Parametres & \multicolumn{2}{|c|}{ RGS } & \multicolumn{2}{c|}{ EPIC-pn } \\
\hline & Comp. 1 & Comp. 2 & Comp. 1 & Comp. 2 \\
\hline$n_{\mathrm{e}} n_{\mathrm{H}} V\left(10^{64} \mathrm{~m}^{-3}\right)$ & $16.78 \pm 0.71$ & $3.51 \pm 0.70$ & $30.10 \pm 10.01$ & $5.34 \pm 3.03$ \\
$k T_{\mathrm{e}}(\mathrm{keV})$ & $0.19 \pm 0.02$ & $0.83_{-0.20}^{+0.05}$ & $0.21 \pm 0.04$ & $0.82_{-0.21}^{+0.40}$ \\
$n_{\mathrm{e}} t\left(10^{16} \mathrm{~m}^{-3} \mathrm{~s}\right)$ & $81.10 \pm 20.10$ & $7.31 \pm 3.11$ & $81.01_{-30.03}^{+80.10}$ & $7.20_{-4.20}^{+10.10}$ \\
\cline { 2 - 5 }$N_{\mathrm{H}}\left(10^{24} \mathrm{~m}^{-2}\right)$ & $9.50 \pm 3.10$ & \multicolumn{2}{|c|}{$12.10 \pm 6.30$} \\
\hline Abundances (wrt solar): & \multicolumn{3}{|c|}{$5.0 \pm 2.12$} \\
\hline $\mathrm{H} / \mathrm{O}$ & $4.96 \pm 1.11$ & \multicolumn{2}{|c}{-} \\
$\mathrm{C} / \mathrm{O}$ & $2.3 \pm 0.82$ & $1.49 \pm 1.02$ \\
$\mathrm{~N} / \mathrm{O}$ & $0.35 \pm 0.21$ & $2.11 \pm 1.32$ \\
$\mathrm{Ne} / \mathrm{O}$ & $1.50 \pm 0.41$ & $1.96 \pm 1.41$ \\
$\mathrm{Mg} / \mathrm{O}$ & $2.03 \pm 1.01$ & $2.01 \pm 1.6$ \\
$\mathrm{Si} / \mathrm{O}$ & $1.78 \pm 1.12$ & $2.64 \pm 1.41$ \\
$\mathrm{~S} / \mathrm{O}$ & - & $2.65 \pm 0.42$ & \multicolumn{3}{|c|}{} \\
$\mathrm{Fe} / \mathrm{O}$ & \multicolumn{2}{|c|}{-} & \multicolumn{2}{|c|}{} \\
\hline
\end{tabular}
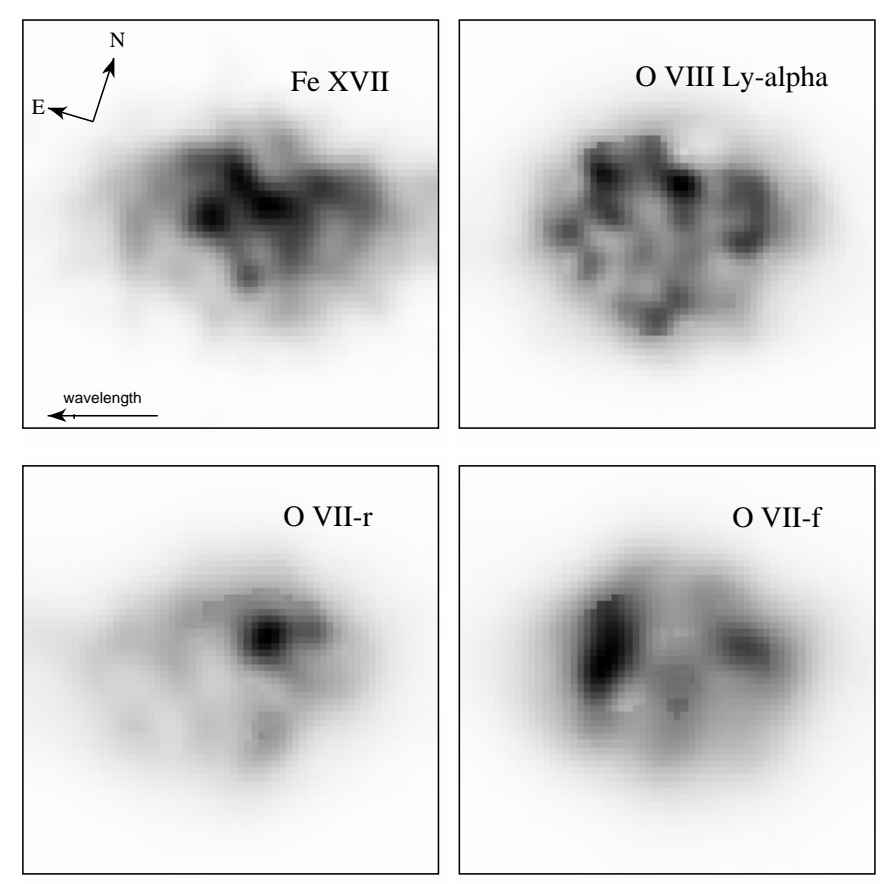

Fig. 4. RGS monochromatic images for Fe XVII (at $17.05 \AA$ ) (upper left) O VIII Ly $\alpha$ (upper right), O VII-resonance (lower left) and O VIIforbidden (lower right) lines. The images are plotted with the dispersion and cross-dispersion on the $X$ and $Y$ axis respectively. North is $18^{\circ}$ clockwise, as indicated. The RGS dispersion direction is such that wavelength increases from right to left in the images.

of the source produces a maximum broadening of $\pm 0.08 \AA$ (O VII-r line), equivalent to a Doppler shift in either direction of $1100 \mathrm{~km} \mathrm{~s}^{-1}$. For comparison, the intrinsic wavelength resolution of the RGS is $\sim 0.06 \AA$ (FWHM) at $21.6 \AA$. However, any Doppler broadening is also convolved along the dispersion direction and, depending on its magnitude, could distort
Table 2. EPIC-pn spectral fitting results for the shell and central region. The abundance values are all normalised to $\mathrm{O}$ and relative to solar (Anders \& Grevesse 1989).

\begin{tabular}{|c|c|c|c|}
\hline \multirow[t]{2}{*}{ Parametres } & \multicolumn{2}{|c|}{ Shell } & \multirow[t]{2}{*}{ Central region } \\
\hline & Comp. 1 & Comp. 2 & \\
\hline$n_{\mathrm{e}} n_{\mathrm{H}} V\left(10^{64} \mathrm{~m}^{-3}\right)$ & $6.11_{-0.31}^{+2.04}$ & $4.52_{-0.22}^{+1.10}$ & $0.65 \pm 0.22$ \\
\hline$k T_{\mathrm{e}}(\mathrm{keV})$ & $0.36_{-1.34}^{+0.12}$ & $0.79 \pm 0.05$ & $1.12_{-0.33}^{+0.11}$ \\
\hline$n_{\mathrm{e}} t\left(10^{16} \mathrm{~m}^{-3} \mathrm{~s}\right)$ & $4.7_{-0.5}^{+30.1}$ & $11.1 \pm 0.22$ & $4.1 \pm 0.8$ \\
\hline \multicolumn{4}{|c|}{ Abundances (wrt solar): } \\
\hline $\mathrm{Si} / \mathrm{O}$ & \multicolumn{2}{|c|}{$1.53 \pm 0.22$} & $2.32 \pm 0.91$ \\
\hline $\mathrm{Fe} / \mathrm{O}$ & \multicolumn{2}{|c|}{$1.61 \pm 0.34$} & $5.94 \pm 2.10$ \\
\hline
\end{tabular}

the RGS images. We present images for the O VIII Ly- $\alpha$, O VIIresonance $(r)$ and $\mathrm{O}$ VII-forbidden $(f)$ line in Fig. 4. We choose these lines because they are the strongest features in the spectrum and because the $\mathrm{O}$ VII line ratios are valuable plasma diagnostics. We also extracted an Fe XVII image for the purpose of investigating the $\mathrm{Fe}$ emission morphology. The images are all on a on $2 \times 2^{\prime}$ size. The images have been smoothed with a gaussian filter (FWHM of 4 " for O VIII Ly- $\alpha$ and 8 " for the rest).

First we turn our attention to the Fe/O emission morphology. The Chandra images (Hughes et al. 2003) suggest that most of the $\mathrm{O}$ emission originates from the outer shell, while the central region is (as compared to the rim) Fe rich. The Chandra observations, however, cannot resolve line blends so these images are possibly contaminated with underlying continuum emission as well as emission from different ion species (e.g. Ne). For this reason we turn our attention to the monochromatic images supplied by the RGS. The RGS O images and the Fe XVII image (see Fig. 4) confirm the Chandra results. The Fe XVII image shows a central filled morphology 


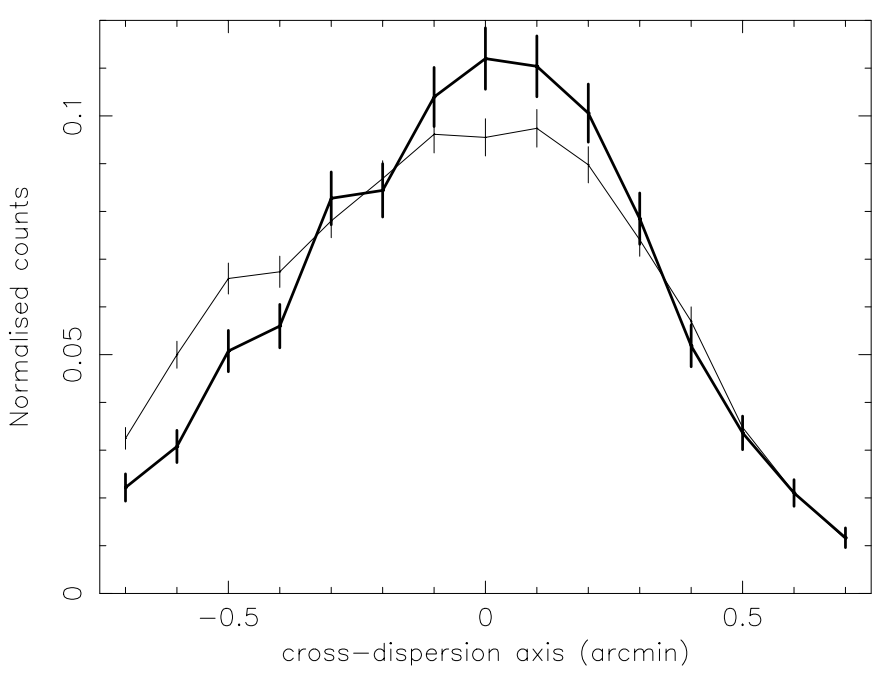

Fig. 5. RGS cross-dispersion profiles for O VIII Ly $\alpha$ (thin solid curve) and Fe XVII (thick solid curve).

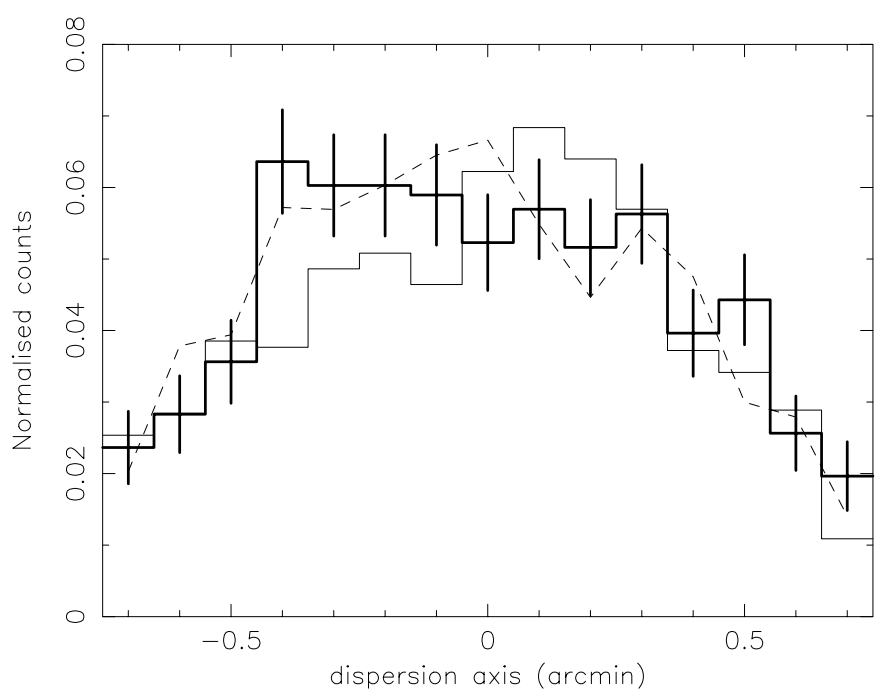

Fig. 6. RGS dispersion profiles for emission lines of O VIII Ly $\alpha$ (dotted curve), O VII-resonance (thin solid curve) and O VII-forbidden (thick solid curve). Error bars are provided for O VII-forbidden line. Errors are typically $50 \%$ and $70 \%$ smaller for the for the O VIII Ly $\alpha$ and $\mathrm{O}$ VII-resonance lines, respectively.

with some emission from the outer region. The $\mathrm{O}$ images show more emission from the outer rim with less emission in the central region. We also examined the cross-dispersion profiles, displayed in Fig. 5, to ensure the statistical significance of these results. We use the cross-dispersion since we have no velocity distortion in this plane, as opposed to the dispersion direction. Here, again, we see that the $\mathrm{Fe}$ emission profile is more centrally peaked as compared to the $\mathrm{O}$ emission.

Next we turn our attention to the differences between the morphology of the O VIII Ly- $\alpha$, O VII $-r$ and O VII- $f$ emission. The images show significant differences between the three lines. The O VIII Ly- $\alpha$ emission predominantly comes from the rim. The O VII- $r$ emission shows a brighter emission spot in the northwestern region while the $\mathrm{O}$ VII- $f$ line shows a bright emission spot on the East rim. To ensure the statistical significance of these results we consider the dispersion profiles,

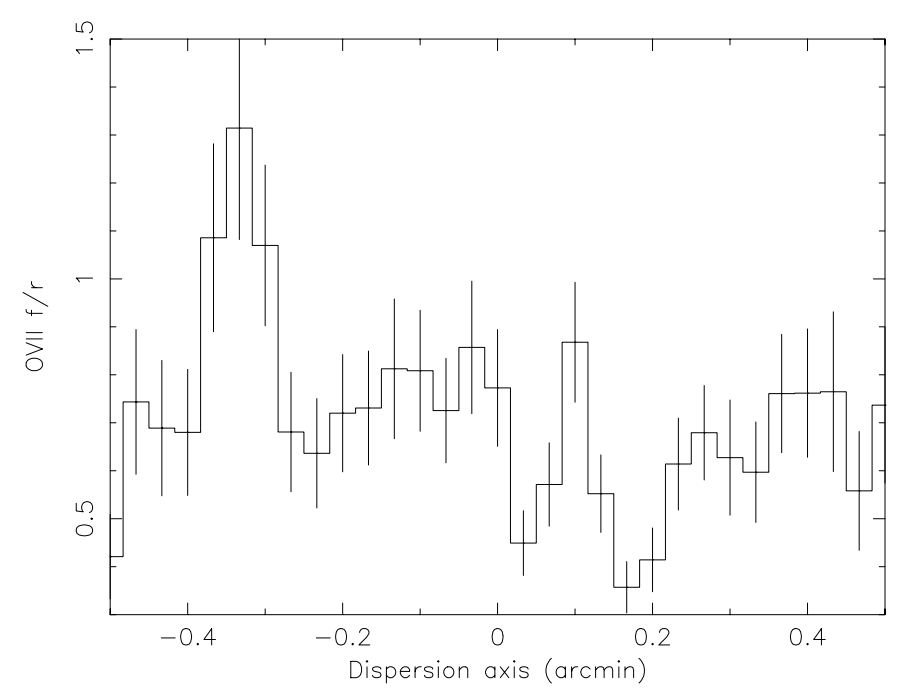

Fig. 7. RGS O VII forbidden/resonance line ratio profile in the dispersion direction.

displayed in Fig. 6. We now use the dispersion profiles because the differences in the lines show up more clearly in this plane. These lines should originate from the same location and have the same velocity structure since they are from the same ion. Any velocity distortion between these images should thus be insignificant as compared to the resolution of our instrument. These profiles confirm the variations in the $\mathrm{O}$ VII- $f$ and $\mathrm{O}$ VII $-r$ images.

One concern could be the possible contamination of O VII $-r$ by its neigbouring O VII- $i$ line. Due to the extent of the source, the O VII- $i$ line emission from the West rim could show up at the long wavelength shoulder of the O VII- $r$ emission line (i.e. the East rim in the O VII- $r$ image). So any possible contamination would cause an increase in the O VII- $r$ line in the East, we instead see the opposite. The intercombination line is, in any case, weak. We checked the spectra from various cross-dispersion regions, but did not see any evidence for an enhanced $i$ line.

\section{Discussion}

\subsection{Single ion spectroscopy}

The O VII $f / r$ line ratios, displayed in Fig. 7, indicate considerable temperature and ionisation age variations in the outer shell. To further investigate the implications of the variations in the monochromatic images we studied the O VII $f / r$ ratio. The ratio plot has a mean value of 0.65 and ranges from $\sim 1.3$ in the East to as low as $\sim 0.4$ in the northwest. In order to understand the implication of these ratios we computed the OVII $f / r$ for a NEI plasma using numerous combinations of temperatures and ionisation ages. The average ratio of $\sim 0.65$ corresponds to a plasma in the temperature range $k T_{\mathrm{e}} \sim 0.1-0.4 \mathrm{keV}$ with $n_{\mathrm{e}} t>3 \times 10^{17} \mathrm{~m}^{-3} \mathrm{~s}$. The ratio of $\sim 0.4$ in the northwest indicates an ionisation age and temperature combination ranging from $\left(n_{\mathrm{e}} t \sim 1 \times 10^{16} \mathrm{~m}^{-3} \mathrm{~s}, k T_{\mathrm{e}} \sim 0.3 \mathrm{keV}\right)$ to $\left(n_{\mathrm{e}} t \sim 1 \times 10^{17} \mathrm{~m}^{-3} \mathrm{~s}\right.$, $\left.k T_{\mathrm{e}} \sim 0.6 \mathrm{keV}\right)$. 
The high $f / r$ value in the East could indicate a low ionisation age $\left(n_{\mathrm{e}} t \sim 1 \times 10^{15} \mathrm{~m}^{-3} \mathrm{~s}\right)$ plasma. However, such a low ionisation age plasma should produce strong O VIIintercombination $(i)$ line emission and we do not see any evidence for this in our data. The observed line ratio can also be obtained if the gas in this region is slowly cooling down to a temperature of $\sim 0.1 \mathrm{keV}$. Such a situation would produce a recombination spectrum with the observed $f / r$ ratio and without a particularly strong O VII- $i$ line. The next question, if we do indeed have a recombining plasma, is the feasibility of cooling in DEM L 71. Recombination effects would become notable if there were some energy draining mechanism operating at the shock front, e.g. particle acceleration (Dorfi \& Böhringher 1993) or radiative shocks. If we assume an initial explosion energy of $E_{0}=1 \times 10^{44} \mathrm{~J}$ and an electron density $n_{\mathrm{e}} \sim 3.2 \times 10^{6} \mathrm{~m}^{-3}$, as obtained in Sect. 4.3, then we estimate (according to Falle 1981) that the radiative cooling phase could commence at an age of $\sim 1.4 \times 10^{4} \mathrm{yrs}$ and this time could be reduced if the blastwave encounters higher density ISM material in this region. If we consider that the estimated age of DEM L 71 is $1 \times 10^{4}$ yrs then this, together with the fact that radiative shocks have also been observed by Ghavamian et al. (2000), makes it possible that we have detected the effects of recombination. We attempted to model the EPIC-pn spectrum from the East rim with a recombination model. This model gave a good description of the data, but required a $\mathrm{Fe}$ abundance of greater than 20 times solar while the other elements had sub-solar abundances. We regard this model as being unphysical. It is probably incorrect to model emission from the entire rim with a recombination model since recombination could only be occurring at a localised region(s) along the rim, as suggested by the bright $f$ line emission spot in Fig. 4 and the results of the Fabry-Perot observations of Ghavamian et al. (2000).

Alternatively, an increase in the $f / r$ line ratio could be introduced by the presence of resonance scattering of the O VII $-r$ photons. In the case of resonance scattering the effective velocity broadening needed to calculate the optical depth is determined from the maximum differential velocity over the region with optical depth $\tau=1$. We estimate a differential velocity of $30 \mathrm{~km} \mathrm{~s}^{-1}$, given an overall expansion velocity of $800 \mathrm{~km} \mathrm{~s}^{-1}$. This differential velocity includes both the gradient in the radial velocity component of the shocked gas as well as the effective azimuthal velocity difference due to the finite (azimuthal) extent of the last scattering surface $\tau=1$. If we assume a uniform spherical shell of thickness $R_{\mathrm{S}} / 12$ and a velocity broadening of $30 \mathrm{~km} \mathrm{~s}^{-1}$, then we could expect an optical depth of $\tau \sim 2.2$ and $\tau \sim 1.0$ in the O VII-r and O VIII Ly $\alpha$ lines, respectively. Following the arguments put forward by Kaastra \& Mewe (1995), an optical depth of $\tau \sim 2.2$ could produce a factor of 2 reduction in the O VII- $r$ line, which would then produce the observed $f / r$ ratio. If the O VII emission originated in a homogeneously filled spherical shell then one would expect that, in the presence of resonance scattering, the O VII- $r$ line image would be more centrally peaked as the photons would escape along the path of shortest optical length. This morphology is not observed. However, the effects of resonance scattering are highly dependent on the morphology and geometry of the

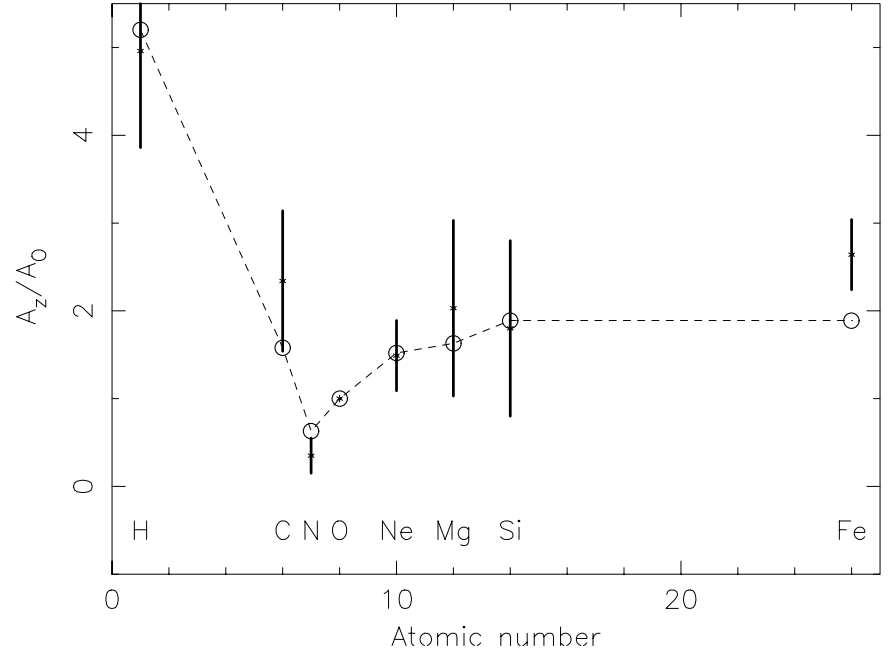

Fig. 8. Elemental abundance ratios as derived from fits to the RGS data. The derived abundance values (crosses) are compared to the average LMC abundance values (open circles). The ratios are all relative to solar (Anders \& Grevesse 1989) and normalised to O. The LMC values are from Hughes et al. (1998).

system and resonance scattering could be limited to localised regions. The observed $f / r$ ratio in the East could come about if the shock front encountered a dense inter-stellar medium (ISM) clump there. The O VII- $r$ photons would then be scattered out of the line of sight, thus producing the observed $f / r$ ratio. We neglected the broadening of the line profile due to microturbulence in the estimation of the optical depth. Microturbulence velocities of a few hundred $\mathrm{km} \mathrm{s}^{-1}$ could reduce the optical depth quite considerably. However, such high microturbulent velocities in DEM L 71 are unlikely.

Further evidence for resonance scattering is provided by the OLine profiles in Fig. 6. Since the estimated optical depth in the O VIII Ly $\alpha$ line is unity, the expected reduction in this line is a factor $\sim 3$ less than for O VII- $r$. The O VIII Ly $\alpha$ and O VII- $f$ line profiles in Fig. 6 are quite similar, while most of the deviation is seen in O VII- $r$. This suggests that the high $f / r$ ratio is brought about by a reduction in the $\mathrm{O}$ VII- $r$ line rather than an enhancement of the O VII- $f$ lines. This is exactly what we would expect in the presence of resonance scattering.

\subsection{Abundances and morphology}

Figure 8 displays the elemental abundance ratios as derived from fits to the RGS data. The abundances are all relative to solar (Anders \& Grevesse 1989) and normalised to O. The derived abundances are also compared to the average LMC ratios (Hughes et al. 1998). The plot shows that the observed abundance values are in good agreement with the LMC values. Most of the emission seen by the RGS arises from the shell. This is clear from the morphology of the monochromatic line images. The shell also dominates the emission in the EPIC images. The outer shell is thus interpreted as being emission from a blast wave which is sweeping up and shock heating ISM material. The idea that the rim emission is from a blast wave heated ISM is supported by the fact that the optical spectrum of DEM L 71 
is Balmer-dominated. Balmer-dominated optical spectra can be interpreted well in the context of a model in which a highvelocity interstellar shock overtakes at least partially neutral interstellar gas (Chevalier \& Raymond 1978). Analysis of the high spatially resolved Chandra ACIS-S CCD image shows that the X-ray outer rim matches the optical $\mathrm{H} \alpha$ image nearly perfectly (Hughes 2001).

The inner region is Fe rich. This is evident from the fits to the EPIC-pn spectra which give $\mathrm{Fe} / \mathrm{O} \sim 1.6$ and $\mathrm{Fe} / \mathrm{O} \sim 6.0$ for the shell and inner regions respectively. This Fe enrichment is also visible in the RGS results, which show a higher $\mathrm{Fe} / \mathrm{O}$ ratio as compared to the LMC value. The higher Fe abundance has also been noted in previous ASCA observations (Hughes et al. 1998). This indicates that the inner shell structure seen in the Chandra images is (reverse shock heated) Fe-rich SN ejecta. Fe-rich SN ejecta in the inner regions of SNRs is typical for remnants produced by type Ia $\mathrm{SN}$ explosion.

\subsection{Mass estimates}

The results of the line ratios show that the two NEI component model used to fit the entire spectrum is highly simplified. These temperature and ionisation age variations in the plasma are probably also the reason why we need at least two components to model the spectra. Despite our simplified model, we can still use the results of the full spectral fits to estimate parameters such as the mean electron densities and emitting masses. We can infer these parameters from the emission measures, but to do this we need to make a volume estimate. The Chandra images show that the X-ray emission from both regions originate from limb brightened shells. The inner shell has an average radius of $\sim 23^{\prime \prime}$ while the outer shell has an average radius of $\sim 37^{\prime \prime}$. We assume that the outer shell has a thickness of $R_{\mathrm{S}} / 12$. We use the radius and shell thickness assumption to estimate the volume, which in turn is used to calculate the electron density and masses. We estimate the outer shell to have an electron density $n_{\mathrm{e}}=3.2 \times 10^{6} / \sqrt{f} \mathrm{~m}^{-3}$ which yields a swept up mass of $M_{\text {shell }} \sim 80 M_{\odot} \sqrt{f}$, where $f$ is the (shell) volume filling factor.

The mass estimate of the ejecta (central) region depends on the amount of $\mathrm{H}$ mixed into the ejecta during its evolution (see Hughes et al. 2003 for complete explanation). We thus, following the example of Hughes et al. (2003), compute the masses for two astrophysically plausible scenarios; 1) We assume that metals are the sole source of electrons 2) assume that a comparable amount (in mass) of hydrogen has been mixed into the metal rich ejecta. We use the volume estimate for a spherically symetric shell. The Chandra images indicate an average radius of $\sim 23^{\prime \prime}$ and a shell thickness of $\sim 11^{\prime \prime}$ for the central region. A pure metal composition yields $1.1 M_{\odot}$ of $\mathrm{Fe}$ and $0.15 M_{\odot}$ of $\mathrm{Si}$, while an admixture of $\mathrm{H}$ yields $0.7 M_{\odot}$ of Fe and $0.1 M_{\odot}$ of $\mathrm{Si}$. These values are similar to and confirm the results of Hughes et al. (2003).

There are observational and theoretical indications that type Ia $\mathrm{SNe}$ are thermonuclear explosion of accreting white dwarfs (e.g. Nomoto et al. 1994), however, the exact binary evolution has not been identified. Nomoto et al. (1997) provided some constraints on the progenitor system from the viewpoint of nucleosynthesis. They presented nucleosynthesis results for various deflagration speeds to constrain the rate of accretion from the companion star. The measurement of the $\mathrm{Si} / \mathrm{Fe}, \mathrm{S} / \mathrm{Fe}, \mathrm{Ar} / \mathrm{Fe}$ and $\mathrm{Ca} / \mathrm{Fe}$ mass ratios would be useful to distinguish the type Ia models (Nomoto et al. 1997). We are only able to derive mass estimates for $\mathrm{Fe}$ and $\mathrm{Si}$. Nomoto et al. (1997) predicts a $\mathrm{Si} / \mathrm{Fe}$ mass ratio of $\sim 0.25$ for the classical (W7) type Ia model and $\sim 0.44$ for a delayed detonation (WDD2) model. The present data do not allow us to discriminate between models. Deeper observations of DEM L 71 could detect $\mathrm{S}$, Ar and Ca emission which would be useful in identifying the type Ia SNe model.

\section{Summary and conclusions}

The high resolution XMM-Newton emission line spectra of DEM L 71 can be fitted with two NEI plasma components. The RGS spectra are dominated by emission from the SNR shell, so the two NEI components needed to fit these spectra indicate a range of temperatures and ionisation ages present in the shell. The temperatures $\left(k T_{\mathrm{e}} \sim 0.2\right.$ and $\left.0.8 \mathrm{keV}\right)$ are consistent with the shock temperature $\left(k T_{\mathrm{s}}=0.11-0.75 \mathrm{keV}\right)$ obtained from optical results (Smith et al. 1994). Temperature and ionisation age variations are also evident from the difference in morphology between the O VII $f$ and $r$ lines. These lines show an anomalously high ratio $(f / r \sim 1.2)$ in the East. This ratio could imply that in this region the plasma is cooling and recombining. However, an alternative, and equally interesting, possibility is that the variation in $f / r$ ratios is due to resonance scattering, which would reduce $\mathrm{O}$ VII- $r$ line emission along lines of sight with a high O VII column density.

The Chandra images reveal a double shock structure in DEM L 71. The EPIC-pn spectral analysis of these two regions indicates that the inner region $\left(k T_{\mathrm{e}} \sim 1.1 \mathrm{keV}\right)$ is hotter than the shell $\left(k T_{\mathrm{e}} \sim 0.2-0.8 \mathrm{keV}\right)$. The abundance ratios of the outer shell is consistent with the average LMC values, while the inner region shows a much higher $\mathrm{Fe} / \mathrm{O}$ abundance ratio. The outer shell has a much higher mass $\left(\sim 80 M_{\odot}\right)$ compared to the inner shell $\left(\sim 2 M_{\odot}\right)$. The image and spectral analysis of DEM L 71 thus suggests that the remnant has a structure consisting of a hot centre surrounded by a cooler shell. This is in agreement with shock models like Truelove \& McKee (2001), which predict a hotter interior. The outer rim represents a blast wave moving out into the ISM, while the interior emission is from reverse-shock-heated Fe-rich stellar ejecta material. The morphology, mass estimates and abundances strongly suggest that DEM L 71 is the result of a type Ia explosion as previously thought. Our analysis also confirms the recent results of Hughes et al. (2003).

DEM L 71 is a good candidate object to study in order to discriminate between the various type Ia supernovae models (e.g. Nomoto et al. 1997). However, the current data do not allow for this. Deeper observations would provide better statistics to study the ejecta material in more detail, particularly the density profile of the ejecta plasma and the possible detection of $\mathrm{Ar}, \mathrm{Ca}$ and $\mathrm{Fe}-\mathrm{K}$ emission. 
Acknowledgements. We thank Ehud Behar for the valuable discussions we had. We also thank the referee J. Ballet for his detailed comments. The results presented are based on observations obtained with XMM-Newton, an ESA science mission with instruments and contributions directly funded by ESA Member States and the USA (NASA). JV acknowledges support in the form of the NASA Chandra Postdoctoral Fellowship grant nr. PF0-10011, awarded by the Chandra X-ray Center. SRON is supported financially by NWO, the Netherlands Organisation for Scientific Research.

\section{References}

Anders, E., \& Grevese, N. 1989, Geochim. Cosmochim. Acta, 53, 197 Bleeker, J. A. M., Willingale, R., \& van der Heyden, K. J. 2001, A\&A, 365, L225

Brickhouse, N. S., Dupree, A. K., Edgar, R. J., et al. 2000, ApJ, 530, 387

Chevalier, R. A., \& Raymond, J. C. 1978, ApJ, 225, L27

Dorfi, E. A., \& Böhringer, H. 1993, A\&A, 273, 251

Falle, S. A. E. G. 1981, MNRAS, 195, 1011

Feast, M., Whitelock, P., \& Menzies, J. 2002, MNRAS, 329, L7

Ghavamian, P., Hughes, J. P., Rakowski, C. E., \& Williams, T. B. 2000, AASM, 197.820 den Herder, J. W., Brinkman, A. C., Kahn, S. M., et al. 2001, A\&A, 365, L7

van der Heyden, K. J., Behar, E., Vink, J., et al. 2002, A\&A, 392, 955

Hughes, J. P. 2001, in Young Supernova Remnants Eleventh Astrophysics Conference, ed. S. S. Holt, \& U. Hwang, AIP Conf. Proc., 565, 419

Hughes, J. P., Hayashi, I., \& Koyama, K. 1998, ApJ, 505, 732

Hughes, J. P., Ghavamian, P., Rakowski, C. E., \& Slane, P. 2003, ApJ, 582, L95

Kaastra, J. S., \& Jansen, F. A. 1993, A\&AS, 97, 873

Kaastra, J. S., \& Mewe, R. 1995, A\&A, 302, L13

Kaastra, J. S., Mewe, R., \& Nieuwenhuijzen, H. 1996, in UV and X-ray Spectroscopy of Astrophysical and Laboratory Plasmas, p. 411, ed. K. Yamashita, \& T. Watanabe (Tokyo: Univ. Ac. Press)

Mewe, R., Kaastra, J. S., \& Liedahl, D. A. 1995, Legacy, 6, 16

Nomoto, K., Hashimoto, M., Tsujimoto, T., et al. 1997, Nucl. Phys. A, 616, 79

Nomoto, K., Yamaoka, H., Shigeyama, S., Kumagai, S., \& Tsujimoto, T. 1994, in Supernovae, ed. S. Bladman, et al. (Elsevier Sci. Publ.), 199

Smith, R. C., Raymond, J. C., \& Laming, J. M. 1994, ApJ, 420, 286

Strüder, L., Briel, U. G., Dennerl, K., et al. 2001, 365, L18

Truelove, J. K., \& McKee, C. F. 1999, ApJS, 120, 299

Turner, M. J. L., Abbey, A., Arnaud, M., et al. 2001, 365, L27 